\title{
"Der Turm« im Kontext der zeitgenössischen österreichischen Dramatik
}

"[D]ie ganze Welt stürzt zusammen", schrieb Hofmannsthal am 26. November 1918 an Ottonie Gräfin Degenfeld. ${ }^{1}$ Drastisch schildert er das massenhafte Sterben an der Grippeepidemie in Wien ebenso wie die Angst vor Arbeiteraufständen, vor Schießereien und Plünderungen, vor freigelassenen Kriegsgefangenen und Kriminellen. Hofmannsthal hatte im k.u.k. Kriegspressequartier den Weltkrieg als Verteidigung der Kultur propagandistisch unterstützt. ${ }^{2}$ Spätestens nach dessen Ende erschien ihm wie vielen seiner Zeitgenossen der Weltkrieg als eine Katastrophe bisher unbekannten Ausmaßes, die alle materiellen wie geistigen Bereiche der Kultur fundamental veränderte. Seine ungeheure Zerstörungskraft kündigte einen Umbruchsprozess an, der zu einer völligen Neukonfiguration der politischen und ökonomischen Kräfteverhältnisse, des individuellen Selbstverständnisses und der kollektiven sozialen Beziehungen führte. Wie Mathias Mayer bemerkt, war dementsprechend für Hofmannsthal die "Konfrontation von Chaos und Ordnung « das zentrale "Problem der Nachkriegszeit«. ${ }^{3}$

In Österreich hatte der Erste Weltkrieg den Untergang des Hauses Habsburg und das Ende des mit ihm verbundenen und durch es legitimierten Staatsgebildes zur Folge. Mehr noch als der Weltkrieg wurde seine Folgezeit, wurden die Aufstände und Revolutionen 1918/19 als traumatisch erfahren. Sie lösten apokalyptische Ängste, aber auch messianische Hoffnungen aus.

Die Situation im Österreich der Zwischenkriegszeit glich in vielem der in Deutschland, wies aber auch Unterschiede auf:

Österreich, von einem Staat mit etwa 51 Millionen Einwohnern zu einem Kleinstaat mit weniger als 6,5 Millionen reduziert, sah sich von den habsburgischen Erblanden abgekoppelt und damit der Übernationalität verlustig, es sah sich aber auch vom Deutschen Reich getrennt, von dem sich - über

1 BW Degenfeld (1986), S. 391.

2 Vgl. Ulrich Weinzierl, Hofmannsthal. Skizzen zu seinem Bild. Wien 2005, S. 62-68.

3 Mathias Mayer, Hugo von Hofmannsthal. Stuttgart, Weimar 1993, S. 72. 
die Parteigrenzen hinweg - ein großer Teil der Österreicher einen Ausweg aus der wirtschaftlichen Misere und zugleich eine Sicherung der nationalen Zugehörigkeit erhoffte. ${ }^{4}$

Das Ergebnis dieses im Friedensvertrag von St. Germain festgeschriebenen Verbots der Vereinigung mit Deutschland war ein in der Sicht vieler Österreicher unerwünschter und nicht lebensfähiger Staat.

Zudem war die Kohäsion zwischen den hauptsächlich katholisch-konservativ geprägten Alpenregionen und der durch moderne kosmopolitische Strömungen und eine stark jüdisch geprägte intellektuelle Elite gekennzeichneten Hauptstadt, in der knapp ein Drittel der Bevölkerung Österreichs lebte, gering. ${ }^{5}$ Die Suche nach einem neuen `Haus Österreich , die Frage der Legitimität des Nachfolgestaates erschien in einer durch unüberwindliche Gegensätze und bürgerkriegsähnliche Zustände gekennzeichneten Zeit gleichermaßen dringlich wie prekär.

Der Staat ist zu Ende: Diese dem »Turm« zugrundeliegende Diagnose entspricht dem Empfinden vieler Österreicher, dem Staat der Ersten Republik fehle die Daseinsgrundlage und -berechtigung. Folgende Argumentation beruht auf drei miteinander verbundenen Thesen: (1) Hofmannsthals "Turm " ist nur vor der Folie der politischen Situation in Österreich nach 1918 adäquat zu verstehen. (2) Daher ist es für sein Verständnis unabdingbar, ihn in den Kontext zeitgenössischer österreichischer Dramatik zu stellen. ${ }^{6}$ (3) Insbesondere Hofmannsthals Auseinandersetzung mit dem Problem der politischen Führung lässt sich im Vergleich mit zeitgenössischen Stücken aus Österreich genauer konturieren. Die Tatsache, dass die ersten Aufführungen des »Turm« 1928 nicht in Österreich, sondern gleichzeitig in München, Hamburg und Würzburg stattfanden, erscheint symptomatisch für die Distanz, die Hof-

4 Wendelin Schmidt-Dengler, Abschied von Habsburg. In: Literatur der Weimarer Republik 1918-1933. Hg. von Bernhard Weyergraf. München, Wien 1995 [= Hansers Sozialgeschichte der deutschen Literatur vom 16. Jahrhundert bis zur Gegenwart 8], S. 483-548, hier S. 483.

5 Vgl. Judith Beniston, Drama in Austria, 1918-45. In: A History of Austrian Literature 1918-2000. Hg. von Katrin Kohl und Ritchie Robertson. Rochester (NY) 2006, S. 21-52, hier S. 21.

6 Wie Robert Pyrah anhand zahlreicher zeitgenössischer Rezensionen herausgestellt hat, spielte besonders das Burgtheater eine wichtige Rolle bei den widersprüchlichen Konstruktionen einer österreichischen Identität in der Ersten Republik und den damit verbundenen identitätspolitischen Auseinandersetzungen. Robert Pyrah, The Burgtheater and Austrian Identity. Theatre and Cultural Politics in Vienna, 1918-38. Oxford 2007. 
mannsthals Stück zur österreichischen Zwischenkriegsdramatik hielt. Gerade weil dieses Abstandhalten ein wichtiges Movens und Merkmal des »Turm« bildet, ist zu dessen angemessenem Verständnis das österreichische Theater dieser Zeit als Hintergrundfolie zu rekonstruieren. Obschon die politische Diagnose, die sich aus dem »Turm« ablesen lässt, ebenso auf die Weimarer Republik gemünzt ist, war die Situation im Österreich der Ersten Republik nach dem Ende der Habsburger Monarchie für Hofmannsthal exemplarisch und symptomatisch auch für die zeitgenössische Lage in Deutschland.

Wie in der Palette möglicher Sichtweisen und Umgangsformen mit der historischen Realität der Ersten Republik »Der Turm« deutlich aus seinem Umfeld heraussticht, soll im Folgenden an vier Paradigmen aufgezeigt werden.

\section{Religion - Theater als Handlungssteuerung}

1927 wurde die Neufassung von Karl Schönherrs »Volksschauspiel in drei Akten« "Der Judas von Tirol« ein großer Bühnenerfolg. ${ }^{7}$ Schönherr behandelt hier einen historischen Stoff: den Verrat des Tiroler Freiheitskämpfers Andreas Hofer an die Franzosen im Jahr 1810. In einer Inversion des Heldennarrativs wird der Verräter, der Knecht Raff, zum Märtyrer, während der Märtyrer der Freiheit Andreas Hofer im Stück abwesend bleibt. Raffls Wunsch, bei den Passionsspielen Christus zu spielen, bleibt unerfüllt, weil diese Rolle einem »erbgsessenen Bauernsohn « 8 vorbehalten ist. Stattdessen muss er den Judas spielen. Er fühlt sich in seine Rolle so sehr ein, dass er nicht mehr zwischen Theater und Realität unterscheiden kann. So verrät er das Versteck Andreas Hofers, erhält die Belohnung und erhängt sich am Ende. Während die Bauernsöhne und die anderen Landarbeiter das Passionsspiel als eine bezahlte Routine betrachten, ist der gedemütigte und an den Rand gedrängte Raffl der einzige Gläubige, der seine Rolle mit Inbrunst spielt.

\footnotetext{
7 Erstfassung 1897. Verfilmungen 1933, 1978, 2006. Schönherr lebte in Wien, von hier aus eroberten seine Stücke deutsche Bühnen, allerdings führte sie eine Tiroler Truppe, die Exl-Bühne, zum Erfolg.

8 Karl Schönherr, Der Judas von Tirol. Volksschauspiel in drei Akten. In: Ders., Bühnenwerke. Hg. von Vinzenz K. Chiavacci. Wien 1967, S. 679-718, hier S. 683.
} 
Der Barocktopos des >Theatrum mundi< wird bei Schönherr psychologisch und gesellschaftskritisch gewendet. Die ausgehöhlten religiösen Riten und biblischen Erzählungen dienen nur mehr dazu, die sozialen Unterschiede zu legitimieren. Im Zuschauer soll Mitleid mit Raffl und Wut gegen die verkrusteten bäuerlichen Hierarchien geweckt werden. Wenn dagegen Olivier in beiden Fassungen des "Turm « die Erlöserfigur Sigismund töten lässt, so entbehrt sein Verhalten solcher Rechtfertigung und bedarf keiner Empathie. Seine Motive sind Ressentiment, Machtgier sowie die Lust an Gewalt und Zerstörung.

Den Vorgang der Veränderung der Spieler durch ihre Rollen legte auch Hofmannsthals Freund Max Mell seinem 1924 im Rittersaal des Landhauses in Graz uraufgeführten "Apostelspiel « zugrunde. "Das Apostelspiel" ist das mittlere Stück einer Trilogie, die "Das Schutzengelspiel« und »Das Nachfolge-Christi-Spiel« umfasst und 1928 bei den Salzburger Festspielen aufgeführt wurde. Zwei junge Kriegsheimkehrer, die an dem "gelbbraunen russischen Militärmantel « ${ }^{9}$ für das zeitgenössische Publikum als Bolschewiken erkennbar waren, kommen auf einen abgelegenen Bauernhof; die beiden wollen die Bewohner dort ermorden und ausrauben. Sie nennen sich Johannes und Petrus und werden von dem naiv gläubigen Bauernmädchen, das sie bewirtet, für Apostel gehalten. Indem sie zur Tarnung dieses Rollenspiel übernehmen, werden sie geläutert und ziehen weiter, ohne ihren Plan durchzuführen. "Das Apostelspiel« ist ein Besserungsstück in der Tradition des Wiener Volkstheaters mit deutlich antisozialistischer Tendenz. Durch das Spielen biblischer Figuren färben diese auf die Spieler ab, die in der Folge der Revolution abschwören. Die Bauern erscheinen als Garanten der Tradition, die auch die Form des Dramas in Knittelversen verbürgen soll.

Wie der Briefwechsel zwischen Hofmannsthal und Max Mell zeigt, trug jener, der diesen schon in der unmittelbaren Nachkriegszeit tatkräftig gefördert hatte, mit seinen Ratschlägen maßgeblich zur Verstärkung der antibolschewistischen Tendenz des Stückes im Zuge seiner Überarbeitung bei. ${ }^{10}$ Durch die Schärfung des Grundkonflikts zwischen Chri-

9 Max Mell, Das Apostelspiel. In: Ders., Gesammelte Werke 1. Wien 1962, S. 129-166, hier S. 137. Vgl. Schmidt-Dengler, Abschied von Habsburg (wie Anm. 4) S. 497.

10 Die erste Version erschien 1923 in den 'Neuen Deutschen Beiträgen`, die zweite 1924 in der Verlagsbuchhandlung Ulrich Moser in Graz, die letztgültige 1926 in der Insel-Bücherei. 
stentum und Bolschewismus wurden die dramatische Spannung und die Bühnenwirksamkeit des Stückes gesteigert. Insbesondere erhält die ressentimentgeladene, destruktive revolutionäre Rhetorik der Figur des Petrus in der von Hofmannsthal angeregten Überarbeitung eine größere Ähnlichkeit zu der des Olivier in der ersten Fassung des »Turm «. ${ }^{11}$

Im »Apostelspiel« wie im »Turm« spielt die Verbindung von Theatralität und Religion eine zentrale Rolle, doch hat diese Koppelung in beiden Stücken eine völlig andere Funktion. Denn wenn bei Mell die zunächst in täuschender Absicht gespielte Rolle der Apostel zur Läuterung und Bekehrung der Spieler führt, ist bei Hofmannsthal das Rollenspiel des Großalmoseniers ein Mittel, dem Gesprächspartner Basilius eine dringliche Handlungsanweisung zu geben und ihn zu läutern. In seinem mit Musik, Chorgesang und Lesung raffiniert inszenierten Auftritt übernimmt der Großalmosenier die Rolle Antonio de Guevaras, des Hofpredigers Karls V., um Basilius zu bewegen, selbst in die Rolle Karls V. zu schlüpfen und freiwillig abzudanken. Das Rollenspiel wird zum Instrument der Machtausübung, bei der sich die Autorität der religiösen Texte mit der des historischen Vorbilds verbindet. Indem sich aber die historische Analogie im Verlauf des Stückes als hinfällig erweist, wird auch die Autorität der biblischen Apokalypse-Erzählung in Frage gestellt.

Eine solche Problematisierung biblischer Prätexte und Präfigurationen artikuliert sich auch in der Verknüpfung von Religion, Theater und Verrat, die "Der Judas von Tirol « und die Großalmosenier-Szene im »Turm« gemeinsam haben. Als Basilius in der Kinderkönigfassung den Großalmosenier, der in der Sicht des Königs einst als sein Ratgeber die Verstoßung des Sohnes zu verantworten hatte, anfleht: »[G]ib uns unser Kind zurück!«, antwortet dieser: »Jetzt sehe ich einen grossen Schauspieler.«12 Dieses Verdikt trifft in gleichem Maße auf den Großalmosenier zu, der in die Rolle des Entsagenden eben in dem Moment schlüpft, als sich der Sturz des Basilius abzeichnet. ${ }^{13}$ Die Handlung und die Figur des »Ju-

Judith Beniston hat Hofmannsthals Einfluss auf die Überarbeitung überzeugend nachgewiesen. Vgl. Judith Beniston, 'Der Wiener aus Hofmannsthak: The Making of Max Mell's Das Apostelspiel. In: MLR 104 (2009), H. 2, S. 472-498.

11 Zur Gegenüberstellung der Versionen des »Apostelspiels« vgl. ebd., S. 484-486.

12 SW XVI.1 Dramen 14.1, S. 51.

13 Chŏng-ae Nam kommentiert die Szene dementsprechend: "Gerade als Basilius' Macht ins Schwanken geraten ist - oder besser gesagt: gerade weil -, verlässt ihn der Geistliche unbarmherzig [...].« (Chŏng-ae Nam, Das Religiöse und die Revolution bei Hugo von Hofmannsthal. München 2010, S. 61) 
das von Tirol« erscheinen hier invertiert. Wird Raffl aus einer Position der Ohnmacht in die Rolle des Verräters gedrängt und mit dem daraus folgenden Selbstmord diese Ohnmacht besiegelt, so ist für den Großalmosenier der Verrat ein Mittel der Machterhaltung. In der dramatischen Ökonomie verkehrt sich dieses Verhältnis abermals: Raffl ist die Zentralfigur, welche mit ihrem Martyrium erst das Martyrium und die Glorifizierung Andreas Hofers ermöglicht. Der Großalmosenier erweist sich dagegen als für den weiteren Verlauf der Handlung bedeutungslos; die Macht der Kirche muss in beiden Versionen des "Turm« einer neuen Führungsmacht weichen.

Das Ende der Bühnenfassung des »Turm» liest sich als theatrale Parodie der biblischen Erlösungsgeschichte, die bei Schönherr und Mell zwar abgewandelt wird, aber als Hintergrundfolie noch Gültigkeit besitzt. Die letzten Worte Sigismunds: »Gebet Zeugnis, ich war da, wenngleich mich niemand gekannt hat ${ }^{14}$ weisen ihn als Postfiguration Christi aus. ${ }^{15}$ Dieses Zeugnis kann aber nichts, das über die Existenz Sigismunds hinausginge, bezeugen. ${ }^{16}$ Das abgewandelte Bibelzitat wird im Kontext der Bühnenhandlung zur Formel, zur reinen Geste ohne Inhalt. Mathias Mayer betont »die Paradoxie von Unverstandenheit und Zeugenschaft, die Unvereinbarkeit von Erkenntnis und bezeugendem Zeichen", die »dem Schluß der zweiten Fassung jede tröstliche Deutungsmöglichkeit« nimmt. ${ }^{17}$ Nur dem Publikum gegenüber kann solche Zeugenschaft eines Martyriums noch Gültigkeit und Wirksamkeit beanspruchen. In der ersten Fassung übernimmt der Kinderkönig die Rolle des Erlösers, der

14 SW XVI.2 Dramen 14.2, S. 220.

15 "Aber ihr werdet die Kraft des heiligen Geistes empfangen, der über euch kommen wird, und werdet in Jerusalem, in ganz Judäa, in Samaria, bis in die entferntesten Gegenden der Erde Zeugen von mir seyn", sind dem Bericht des Evangelisten Lukas zufolge (Apg 1,8) die letzten Worte des Herrn an seine Jünger, bevor er in den Himmel auffährt. Die Heiligen Schriften des Alten und Neuen Testamentes. Übersetzt und hg. von Leander van Eß. Sulzbach 1877, Neues Testament, S. 153. Im Johannes-Evangelium (Joh 1,10) heißt es: "Er war in der Welt; und die Welt ist durch ihn erschaffen; doch erkannte die Welt ihn nicht." (Ebd., S. 118); ferner Joh 15,26f.: »Wenn übrigens jener Tröster, den ich euch vom Vater herabsenden werde, kommen wird, (nämlich der Geist der Wahrheit, der vom Vater ausgeht) so wird dieser von mir Zeugnis geben. Und auch ihr werdet zeugen, weil ihr vom Anfange her bei mir waret.« (Ebd., S. 144)

16 Christoph König zufolge kann der Satz "nur noch als unangemessener Anspruch auf einen Eigennamen gedeutet werden«. Christoph König, Hofmannsthal. Ein moderner Dichter unter den Philologen. Göttingen 2001, S. 335.

17 Mathias Mayer, Der Turm. In: Kindlers Neues Literaturlexikon, Hugo von Hofmannsthal. Bd. 7. München 1990, S. 1019-1021, hier S. 1020.

274 Roland Innerhofer 
die Verheißung Sigismunds erfüllt. Auf die Verkündigung des Kinderkönigs: "In der Zeit könnet ihr diesen nicht messen: aber ausser ihr, wie ein Sternbild« antwortet das Volk: "Sigismund! bleibe dein Name bei uns! «18 In der Bühnenfassung ist dagegen die analoge Anrufung Sigismunds als charismatischen Erlöser durch "Stimmen", die vom Burghof aus rufen: "[V]erlasse uns nicht!,$^{19}$ ein trügerisches Schauspiel, durch das Sigismund in die tödliche Falle gelockt wird. Anton erkennt dies: "Recht komödiantisch gebärden sich die. Das sind keine ehrlichen Leut. $\ll^{20}$ Sigismund dagegen vertraut seiner charismatischen Wirkung, welche Oliviers Gefolgsleute inszenieren, und wird so zum Opfer von Oliviers Scharfschützen. Das apokalyptische Ende der Bühnenfassung ist des Heils verlustig gegangen, es beruht auf einer Farce.

Deutlich ist der Abstand, der die Bühnenfassung des "Turm« von Mells Konzept einer Verbesserung des Menschen durch das Spielen einer guten Rolle trennt. Wie die beiden Aposteldarsteller lernt Sigismund im Laufe eines Tages, an dem er König spielt, den Gewaltverzicht. Bei Mell sind es die Vertreter des Bolschewismus, die durch die Begegnung mit dem unverdorbenen gläubigen Mädchen zum Verzicht auf revolutionäre Gewalt bekehrt werden. Hofmannsthals Olivier wird zwar ebenfalls als Bolschewik, ${ }^{21}$ der seine Gewaltherrschaft durch einen vorgeblichen Volksauftrag legitimiert, gekennzeichnet, ist aber wider solche Konditionierung durch Rollenspiel immun. Als Techniker der Macht will er sich des Schauspiels bedienen, um die Masse zu manipulieren. Der vom Volk verehrte Sigismund soll wie ein Idol ausgestellt werden, um die Wut der Masse gegen feudales Unrecht anzustacheln. Die Weigerung, diese Rolle zu übernehmen, bezahlt Sigismund mit dem Tod; ein Doppelgänger wird seinen Part übernehmen. Während also Mell die Revolutionäre gerade auch durch die sie verwandelnden Rollen individualisiert, sind

18 SW XVI.1 Dramen 14.1, S. 139.

19 SW XVI.2 Dramen 14.2, S. 219.

20 Ebd., S. 220.

21 In den Erläuterungen zur Entstehung der Bühnenfassung des »Turm» (SW XVI.2 Dramen 14.2, S. 239-241) wird das Buch von René Fülöp-Miller, Geist und Gesicht des Bolschewismus. Darstellung und Kritik des kulturellen Lebens in Sowjet-Russland. Zürich, Leipzig, Wien 1926, als Quelle für die Darstellung Oliviers nachgewiesen. Nicht nur mehrere Selbstzeugnisse Hofmannsthals legen diese historische Signatur der Figur Oliviers nahe, auch die zeitgenössische Rezeption verstand Olivier als Bolschewiken. Vgl. Marcus Twellmann, Das Drama der Souveränität. Hugo von Hofmannsthal und Carl Schmitt. München 2004, S. 139,141 und $148 f$. 
in der Bühnenfassung des "Turm" die erfolgreichen Führer der Masse Typen und Masken. So muss Sigismund, der durch seine exzeptionellen und vorbildlichen individuellen Qualitäten den charismatischen Führer verkörpert, Olivier weichen - dem Werkzeug einer historischen Mechanik, die keine autonomen Akteure, sondern bloß austauschbare Komparsen kennt. "Sigismund, die Kreatur einer vollkommenen immanenten Transzendenz [...], wird vom kühl kalkulierenden Machttechniker hinweggeräumt. «2 $^{22}$ Fortan wird die charismatische Wirkung, auf die Olivier angewiesen bleibt, von einer Fälschung ausgeübt, das Idol von der Person losgelöst. So ist die von Olivier geplante Ausstellung eines Doppelgängers auch Parodie auf die symbolische Strahlkraft eines geistigen Führers, der die amorphen Volksmassen organisieren und zu einer Einheit synthetisieren soll.

Sigismund kann in seiner Sprache und in seiner Haltung des Suchenden als Figuration des in der Rede "Das Schrifttum als geistiger Raum der Nation" vorgestellten Führers, der die Gegensätze von Geist und Leben, Individuum und Gesellschaft versöhnt und darin eine Nähe zum Künstler aufweist, verstanden werden..$^{23}$ Die Bühnenfassung des »Turm« zeigt aber gerade die Gefahren eines solchen Projekts kunstaffiner geistiger Führerschaft: Olivier bedient sich eben dieses Konzepts, um seine diktatorische Macht propagandistisch abzustützen: »Olivier not only represents a political figure but can also be seen as an artist, inasmuch as he understands and wields the magic power of symbols. $\aleph^{24}$ Olivier geht es nicht darum, die Masse zu einer geistigen Gemeinschaft zu vereinen, sondern sie - unter dem Vorwand angemaßter Vertretung der Volksinteressen und unter Vorspiegelung eines Vollzugs des Volkswillens gezielt zu manipulieren und zu steuern. Der künstlerische, suggestive Umgang mit Zeichen und Symbolen ist zum Instrument der politischen

22 Alexander Mionskowski, Souveränität als Mythos. Hugo von Hofmannsthals Poetologie des Politischen und die Inszenierung moderner Herrschaftsformen in seinem Trauerspiel »Der Turm« (1924/25/26). Wien 2015, S. 484.

23 Zum Zusammenhang zwischen dichterischem, kulturellem und politischem Führertum in der Schrifttumsrede und im »Turm« vgl.: Twellmann, Das Drama der Souveränität (wie Anm. 21), S. 203-225. Schon William H. Rey hat zu Recht betont, dass die in der Schrifttumsrede entworfene Vision geistiger Führerschaft in beiden Versionen des "Turm" als problematisch erscheint. William H. Rey, Hofmannsthal: Der Turm. In: Das deutsche Drama. Bd. 2: Vom Realismus bis zur Gegenwart. Düsseldorf 1964, S. 267-285.

24 Hang-Sun Kim, »Unser Dasein starrt von Büchern«: Hugo von Hofmannsthal's Crisis of Authorship. Doctoral dissertation, Harvard University 2012, S. 163, online unter http:// nrs.harvard.edu/urn-3:HUL.InstRepos:9793868 [Zugriff: 15.11.2016]. 
Täuschung, Verdunkelung und Realitätsverzerrung verkommen. Die Gewaltherrschaft, die sich metaphysisch als »Fatalität« bemäntelt, bedarf gleichwohl magischer und ritueller Praktiken, der religiösen Aura einer charismatischen Führergestalt.

\section{Masse und Gewalt oder Geistige versus soziale Revolution}

Die Relation von Macht und Gewalt ist in Hofmannsthals "Turm" verschränkt mit der Spannung zwischen den Konzepten einer geistigen und einer sozialen Revolution. Dieser Konflikt ist auch in der zeitgenössischen österreichischen Dramatik von zentraler Bedeutung.

1923 wurde postum Hans Kaltnekers Drama »Das Bergwerk" als tausendste Vorstellung der sozialdemokratischen Kunststelle im Wiener Raimundtheater uraufgeführt. ${ }^{25}$ Kaltneker, der 1919 an Tuberkulose starb, stand Hofmannsthal nahe, er führte dessen lyrische Dramen privat mit Freunden auf.

"Das Bergwerk « ist ein Revolutions- und Verkündigungsdrama, das einer expressionistischen »kritischen Mitleidsdramatik « ${ }^{26}$ zugeordnet werden kann. Die Zeitangabe »Dezember eines Jahres im 20. Jahrhundert « ${ }^{27}$ verweist unmissverständlich auf die Novemberrevolution 1918/19 in Deutschland und Österreich. Verschüttet in einer Mine macht der Held Michael angesichts des Todes die mystische Erfahrung der Güte alles Lebenden. Der Arbeiterführer verwandelt sich vom Revolutionär zum Friedliebenden, der das Evangelium der Nächstenliebe verkündet. Er weigert sich, die Grubenarbeiter in einen Generalstreik gegen ihre Unterdrücker zu führen und wird deshalb von einem jüngeren Mann erschossen. Dieser war zusammen mit ihm verschüttet, verlor dabei seinen Vater und ist seither von Rachegelüsten erfüllt. Die Handlung entspringt der Spannung zwischen gewaltsamer sozialer und friedlicher geistiger Erneuerung. Politisches und Privates widersprechen einander: Michael

25 Der 1895 in Temesvár geborene, 1906 mit seiner Familie nach Wien gekommene Kaltneker hieß eigentlich Hans von Wallkampf.

26 Margret Dietrich, Österreichische Literatur (\$§ 16-25). In: Reallexikon der deutschen Literaturgeschichte. Hg. von Werner Kohlschmidt und Wolfgang Mohr. Bd. 2. Berlin, New York ${ }^{2} 2001$, S. 753-765, hier S. 764.

27 Hans Kaltneker, Das Bergwerk. Ein Drama in drei Akten. In: Ders., Dichtungen und Dramen. Hg. von Paul Zsolnay. Eingel. von Felix Salten. Berlin, Wien, Leipzig 1925, S. 157249, hier S. 159. 
wird nach seiner Befreiung aus dem verschütteten Stollen nach zwanzigjähriger kinderloser Ehe ein Sohn geboren. In der Folge ist er innerlich gespalten: zwischen dem Bedürfnis, durch die soziale Umwälzung die Lebensbedingungen seines Sohnes zu verbessern, und der moralischen Überzeugung, dass aus Gewalt keine Liebe entstehen kann. Michaels Verwandlung vom Verfechter des bewaffneten Aufstands zum Dulder und Märtyrer wird durch ein Erlebnis ausgelöst, dem auch in Hofmannsthals zwei Jahre zuvor uraufgeführtem Lustspiel »Der Schwierige« eine entscheidende Bedeutung zukommt: Durch das Verschüttet-Werden fällt die Hauptfigur aus der Zeit, der sie fortan fremd, aber auch souverän gegenübersteht. Die individuelle mystische Erleuchtung verspricht eine Wende zur kollektiven metaphysischen Erlösung.

Hofmannsthals Sigismund ist anders als Kaltnekers Michael eine gebrochene Figur. Sigismund lernt zwar in der Bühnenfassung durch den Gewaltausbruch während der theatral inszenierten >Prober in der Königsburg den Gewaltverzicht, geht aber daraus nicht als Erleuchteter hervor. In beiden Dramen führt der Gewaltverzicht des Führers, der von der Masse verehrt wird, zu seiner gewaltsamen Beseitigung. Doch Michael opfert sich bewusst, während der getäuschte Sigismund zum passiven Opfer wird.

Sigismund ist politisch ohnmächtig: Weder mit noch ohne Gewalt vermag er seine Ideale zu verwirklichen. Dieses Problem der fehlenden legitimen Macht ist auch grundlegend für das zeitgenössische historische Drama. Als exemplarisch kann Franz Werfels 1924 erschienenes, 1925 uraufgeführtes Stück »Juarez und Maximilian« angesehen werden. ${ }^{28}$ Wie "Der Turm" so besaß auch diese "dramatische Historie in 3 Phasen und 13 Bildern" einen unmittelbaren aktuellen Bezug in der Abdankungsthematik..$^{29}$ Der 1922 verstorbene letzte österreichische Kaiser, Karl I., hatte sich geweigert, formell abzudanken - ebenso wie der von Karl V. abstammende Kaiser Maximilian von Mexiko, den diese Weigerung im Stück das Leben kostet. Im "Turm« ist das Abdankungsmotiv in zwei Varianten wirksam: Basilius wird in der Bühnenfassung zum Abdanken gezwungen, es handelt sich also um keine Abdankung im eigentlichen Sinn. Sigismund dagegen verzichtet freiwillig auf die Königsmacht, da er

28 Im Magdeburger Stadttheater uraufgeführt. Am 26. Mai 1925 gab es Max Reinhardt im Wiener Theater in der Josefstadt, wo es ein großer Erfolg wurde.

29 Darauf verweist Beniston, Drama in Austria (wie Anm. 5), S. 42.

278 Roland Innerhofer 
sich weder von seinem Vater Basilius noch von seinem Erzieher Julian noch vom Rebellenführer Olivier für ihre Zwecke benutzen lassen will. Für die Kinderkönigfassung gilt Mayers Feststellung: Sigismund »dankt von der Macht und vom Leben ab und beweist darin seine Souveränität, denn diese Abdankung verwahrt ihn gegen die Instrumentalisierung durch die Mächtigen. $\aleph^{30}$ In der Bühnenfassung ist »eben diese Gewissheit wieder zurückgenommen «, ${ }^{31}$ mit der geplanten Ersetzung des ermordeten Sigismund durch einen Doppelgänger scheint sich Olivier dessen Charisma dienstbar machen zu können; der Machtverzicht Sigismunds bleibt damit folgenlos.

In Werfels Stück übernimmt Maximilian genau jene Rolle, die Hofmannsthals Sigismund ablehnt: Er wird zur Marionette eines Mächtigen. Dabei ist der Antagonist Benito Juarez nicht auf der Bühne zu sehen. Diesen Kunstgriff des abwesenden Protagonisten hat Werfel, wie weiter unten ausgeführt wird, schon in "Bocksgesang" angewendet. Das Schauspiel stellt den gutgläubigen und gutwilligen Maximilian in den Mittelpunkt, der ohne direkte Konfrontation mit Juarez Opfer der Machtpolitik seines unheimlich gesichtslosen Gegenspielers wird. Axel Schalk betont die Fremdbestimmung Maximilians: "Juarez ist das Synonym für Macht, die als szenisch nichtpräsente Instanz alles bewegt. « ${ }^{32}$ Ob Maximilian aber, wie Schalk meint, nur Instrument eines abstrakten "Geschichtsautomatismus « ${ }^{33}$ ist, bleibt im Stück unentschieden. Hans Wagener gibt zu bedenken, dass Maximilian auch an seinem selbstverantworteten unzeitgemäßen und inkonsistenten Führungsstil, der in scharfem Kontrast zu dem des Juarez steht, scheitert: Maximilian ist "a ruler who wants to do good but who fails because history has surpassed him and his beliefs $-[. .$.$] who meant well and became guilty; who had$ betrayed his ideas out of personal weakness and hurt pride. $\aleph^{34}$

30 Mathias Mayer, Die Kunst der Abdankung. Neun Kapitel über die Macht der Ohnmacht. Würzburg 2001, S. 120.

31 Ebd.

32 Axel Schalk, Franz Werfels Historie "Juarez und Maximilian«. Schicksalsdrama, "neue Sachlichkeit« oder die Formulierung eines paradoxen Geschichtsbilds? In: WW 38, 1988, S. 78-87, hier S. 83.

33 Ebd.

34 Hans Wagener, Understanding Franz Werfel. Columbia (SC) 1993, S. $56 f$. 
Maximilian wird in seinem idealistischen Glauben an die eigene Mission auch mit seinem Bruder Franz Joseph kontrastiert. Doch Maximilians Selbststilisierung als "Stellvertreter der weltlichen Liebe Gottes « ${ }^{35}$ ist kaum geeignet, die Legitimität monarchischer Herrschaft zu restituieren: "Die souveräne Epoche ist vorüber. [...] Die Zeit der Diktatoren beginnt« ${ }^{36}$ äußert Maximilian selbst. Juarez ist ein nüchterner Techniker der Macht, der sich zu ihrer Erlangung der Masse zu bedienen weiß. Wie sich Olivier in der Bühnenfassung selbst inszeniert, so wird Juarez von seiner Entourage wahrgenommen: als Instrument historischer Fatalität. »Don Juarez ist die schlichte Vernunft selbst« ${ }^{37} \mathrm{er}$ »hat nie einen Traum geträumt«. ${ }^{38}$

Werfel bringt der in Maximilian verkörperten Kaiseridee durchaus eine gewisse Sympathie entgegen, und zwar obwohl oder gerade weil Maximilian, wie Wynfrid Kriegleder formuliert, »die träumende Unvernunft" ist; "er tut nicht das Richtige, sondern das Gute. «39 Das Scheitern des Versuchs, die Legitimität monarchischer Herrschaft durch die Volksmassen neu zu begründen, verbindet auch Maximilian und Sigismund. Doch während Maximilian den Kontakt zu den Massen nicht herstellen kann, wird Sigismund in der Bühnenfassung gerade die Verehrung, die ihm die Armen bezeugen, zum Verhängnis. Denn als sich Sigismund weigert, sich von Olivier zum Zweck der Massenmanipulation benutzen zu lassen, wird er von diesem beseitigt und durch ein Double ersetzt.

\section{Der Ausgestoßene als Aufrührer}

Ein bühnenwirksames Beispiel für die kritische Auseinandersetzung mit der proletarischen Revolution und der Natur politischer Führerschaft nach dem historischen Ende der Monarchien ist Franz Werfels »Bocks-

35 Franz Werfel, Juarez und Maximilian. Dramatische Historie in drei Phasen und dreizehn Bildern. In: Ders., Gesammelte Werke. Die Dramen. 1. Bd. Frankfurt a.M. 1959, S. 385-465, hier S. 414.

36 Ebd., S. 459.

37 Das äußert der Lizentiat Elizea, der Sekretär des Präsidenten Juarez (ebd., S. 395).

38 So seine Charakterisierung durch Riva Palacio, General der rechtmäßigen Regierung unter Don Benito Juarez (ebd., S. 423).

39 Wynfrid Kriegleder, Der Legitime und der Republikaner: Franz Werfels »Juarez und Maximilian«. In: Unser Fahrplan geht von Stern zu Stern. Zu Franz Werfels Stellung und Werk. Hg. von Joseph P. Strelka und Robert Weigel. Bern u.a. 1992, S. 165-176, hier S. 167. 
gesang" aus dem Jahr $1921 .{ }^{40}$ Hier wird ein von den Eltern versteckter deformierter Sohn zur Symbolfigur des Aufstands der sozial Ausgestoßenen in einer slawischen Bauerngesellschaft. ${ }^{41}$ Der Titel »Bocksgesang« bezeichnet die deutsche Entsprechung des griechischen Wortes $\tau \rho \alpha \gamma \omega \delta i ́ \alpha$. Die Bezugnahme auf die Riten zu Ehren des Weingottes Dionysos, in denen der Ursprung der tragischen Gattung lag, deutet schon den $\mathrm{Zu}$ sammenhang an, in den Werfels Drama Exklusion und Führung stellt.

Wie im » Turm " wird auch in "Bocksgesang « eine ödipale Konstellation politisch gedeutet: Im Unrecht gegen den weggesperrten Sohn erkennen diejenigen, denen Grundbesitz verwehrt wird - repräsentiert durch einen Juden, einen Remigranten aus Amerika und einen Schauspieler - ihre Lage wieder. Der seinem Gefängnis entkommene Bock wird zu ihrem Rächer. Er verkörpert eine entfesselte Triebhaftigkeit und Vitalität, wie sie beispielhaft in Arnolt Bronnens expressionistischem Stück "Vatermord « (1920) zum Tragen kommt. ${ }^{42}$ Der Vatermord geht bei Bronnen mit einer Verherrlichung der Jugend einher, die sich von der "Fessel - so der sprechende Name des Vaters - der Vätergeneration und ihrer Werte befreien muss. Wie Friedbert Aspetsberger zeigt, fehlt den "jungen Menschen« in der Literatur der Zwischenkriegszeit »der von ihnen selbst gesicherte und daher vom Vater nicht zu gefährdende Lebensraum, der Platz am Licht, das auch auf die anderen fällt, und nicht der Platz im Schatten oder unter den Füßen des Vaters. « ${ }^{43}$

Eben solche Enge und solcher Lichtmangel kennzeichnen im »Turm« den Raum, in dem Sigismund auf Befehl des Vaters eingesperrt ist. ${ }^{44}$ Wenn das Thema der neuen Macht auch im »Turm" mit einem Generationenwechsel verbunden ist, so äußert sich dieser im Ausbruch

40 Das Stück wurde 1922 im Wiener Raimund-Theater unter der Regie von Rudolf Beer uraufgeführt.

41 "Die Handlung spielt in einer slawischen Landschaft jenseits der Donau an der Wende des achtzehnten zum neunzehnten Jahrhundert.« Franz Werfel, Bocksgesang. In fünf Akten. In: Ders., Die Dramen. 1. Bd., S. 251-317, hier 252.

42 Das Stück wurde am 22. April 1922 im Frankfurter Schauspielhaus unter der Regie von Wolfgang Harnisch uraufgeführt. Die Berliner Erstaufführung fand am 14. Mai 1922 auf der Jungen Bühne im Deutschen Theater unter der Regie von Berthold Viertel statt. Vgl. Arnolt Bronnen, Werke. Mit Zeugnissen zur Entstehung und Wirkung hg. von Friedbert Aspetsberger. Bd. 1. Klagenfurt [1989], S. 368f.

43 Friedbert Aspetsberger, rarnolt bronnen‘. Biographie. Wien, Köln, Weimar 1995, S. 211.

44 "Ich habe meinen einzigen Sohn von mir getan, - dahin wo ihn die Sonne nicht bescheint!«, so Basilius in beiden Fassungen zum Großalmosenier. SW XVI.1 Dramen 14.1, S. 48; SW XVI.2 Dramen 14.2, S. 43. 
aus einem eingeengten Raum, einem Familiengefängnis. Anders als in Bronnens Stück, in dem sich der Sohn aus eigener Kraft aus dieser Enge befreit, setzt sich Sigismund zwar im dritten Akt durch jugendliche Körperstärke und Tatkraft gegen den Vater als physische Person durch, nicht aber gegen dessen Befehlsgewalt: Er wird von den dem König loyalen Kämmerern überwältigt. Während der Kampf zwischen Vater und Sohn in "Vatermord« in einem proletarischen Milieu stattfindet und einzig durch Körperkraft entschieden wird, ist der Kampf zwischen Basilius und Sigismund in das System dynastischer Filiationen, feudaler Loyalitäten und Legitimationen eingebunden. Und während Bronnens Stück das Sexuelle verabsolutiert und daher politisch nicht festlegbar ist, sind die Körper bei Hofmannsthal immer auch Repräsentanten von Positionen im Machtgefüge, die körperlichen Beziehungen und Interaktionen von den historisch jeweils dominanten Regeln des politischen Handelns geprägt. Dementsprechend bleibt die Lehre, die Sigismund in der Bühnenfassung aus der vorerst gescheiterten Entmachtung des Vaters zieht, nämlich die Konzentration auf seine geistige Kraft, politisch wirkungslos; sie ist unzeitgemäß, da sie an keine politische Kraft andocken kann. Entmachtet wird der König von Olivier, der den plebejischen Aufstand entfesselt und dessen rohe Gewalt gezielt einsetzt.

In "Bocksgesang" wird der Sohn in zwei Kontrastfiguren aufgespalten. Dem wohlgestalteten, aber schwachen Sohn steht der missgestaltete, aber vitale gegenüber. Dieser übt auf Frauen wie Männer eine geheime Anziehungskraft aus. Der Student, der bei Werfel, wie der ehemalige Student Olivier im »Turm", als Sprecher der Ausgestoßenen figuriert, hat den Bock gefangen und in einer Kirche eingesperrt. Hier steigert sich die Feier und Verklärung des Dionysos-Bockes zur orgiastischen Ekstase. In den Bühnenanweisungen heißt es: »Die Weiber schütteln die gelösten Haare und beginnen, die Kleider zu zerreißen « ${ }^{45}$, »die Weiber brechen in Schreie, die Männer in keuchende Rufe aus « ${ }^{46}$.

45 Werfel, Bocksgesang (wie Anm. 41), S. 295.

46 Ebd., S. 297.

282 Roland Innerhofer 
Werfels Darstellung der Massenerregung und -ekstase erinnert an die Funktionsbeschreibung der Phänomene kollektiver Selbstentgrenzung in Sigmund Freuds Schrift "Massenpsychologie und Ich-Analyse (1921). ${ }^{47}$ Bei Werfel werden die von Freud betonten sprimitiven menschlichen Triebe zum Motor eines kollektiven Rituals. Am Schluss des Aktes opfert sich die Verlobte des wohlgeratenen Sohnes dem missratenen. Die neue Zeit, so suggeriert das Stück, beginnt mit der Vereinigung mit dem Tier, bei der das Tier im Menschen befreit und der Mensch als Tier erlöst werden soll. Analog zum "Turm« wird auch in "Bocksgesang" die Revolution mit einem Familiendrama verquickt, ihr Führer ist ein Mensch, dessen Existenz verheimlicht und der wie ein Tier eingesperrt wurde. Sigismund weist darin Parallelen zum werfelschen Bock auf. Bei Werfel ist allerdings nicht die Prophezeiung, sondern die Missgestalt des Sohnes der Grund für seine Verstoßung. Der Krüppel, die Symbolfigur der Revolution, bleibt auch nach seiner Befreiung unsichtbar. Während die Bühnenfassung des "Turm" nach dem Tod Sigismunds keine andere Perspektive als die Herrschaft der »Fatalität $^{48}$ eröffnet, ist am Ende von »Bocksgesang«, nachdem das Monster im brennenden Wald verschwunden ist, Stanja, die Verlobte seines Bruders, von dem Missgestalteten schwanger: In der Latenz kündigt sich eine Erlöserfigur an - und die Wiederkehr des Monsters: »Wenn Stanjas Opfer zur Zeugung eines neuen Monstrums führt, dann ist die kathartische Lösung der sozialen Konflikte, die der Schlussakt zunächst andeutet, in der Allusion einer katastrophischen Zukunft dekonstruiert. « ${ }^{49}$

\section{"Zu früh dran«}

\section{Generationenwechsel und Märtyrertum}

Der Ausgestoßene als Garant der Erneuerung und seine Rückkehr als Versprechen sind auch in Friedrich Schreyvogls historischen Habsburger-

\footnotetext{
47 Darauf verweist Peter-André Alt, Katharsis und Ekstasis. In: Die Tragödie der Moderne. Gattungsgeschichte - Kulturtheorie - Epochendiagnose. Hg. von Daniel Fulda und Thorsten Valk. Berlin, New York 2010, S. 177-205, hier S. 199 f.

48 SW XVI.2 Dramen 14.2, S. 215.

49 Alt, Katharsis und Ekstasis (wie Anm. 47), S. 203.
} 
dramen handlungsbestimmend. ${ }^{50}$ In seinem als "österreichische Ballade« bezeichneten, $1926 \mathrm{im}$ Theater in der Josefstadt in Wien uraufgeführten Drama "Johann Orth" ist Erzherzog Johann Salvator, der 1889 seinen Titel aufgab, um die Bürgerliche Ludmilla (»Milli«) Stubel zu heiraten, Sprachrohr einer idealisierten Österreichvision und Repräsentant eines romantischen Erlösungsgedankens.

Führerschaft beruht bei Schreyvogl auf geistiger Auserwähltheit. Die Hoffnung auf politische Erneuerung wird mit einem Generationenwechsel verbunden. Diese Erneuerung muss in »Johann Orth" scheitern, weil sie in der Person Johanns "zu früh dran « ${ }^{51}$ ist. Auch Sigismund kann als ein Herrscher gesehen werden, dessen Zeit noch nicht gekommen ist. Doch anders als in der Bühnenfassung des "Turm« ist bei Schreyvogl der Geist langfristig mächtiger als die (Tages-)Politik. Das Martyrium dessen, der die "österreichische Idee « ${ }^{52}$ verkörpert, garantiert ihren Fortbestand. Sie ist zugleich die Idee des "großen Deutschen Reiches $\aleph^{53}$ und seines kulturellen Auftrags, den Osten zu kolonisieren.

In Schreyvogls Überarbeitung von "Johann Orth", die 1933 unter dem Titel "Habsburgerlegende« erschien und im selben Jahr mit größerem Erfolg am Burgtheater uraufgeführt wurde, gesellt sich zu Johann Salvator Kronprinz Rudolf als Sprecher einer Österreichidee, die sich auf das Christentum stützt und auf das Heilige Römische Reich Deutscher Nation zurückgreift. Die »Legende« besteht in der Rückkehr Johanns ins republikanische Österreich, wo er in effigie, als sprechende Wachsfigur erklärt: "Der Traum von Johann Orth lebt, nicht er. ${ }^{54}$ In beiden Fassungen ist der Entmachtete und Ausgewanderte als Träger einer dauerhaften Idee politisch wirksam. Der 'Traum vom Reich ist wirklicher als die republikanische Wirklichkeit der Gegenwart. ${ }^{55}$ Erzherzog Johann bezeichnet in einem Gespräch mit Kronprinz Rudolf Deutschland als »Herz" Europas $^{56}$ und in einem Dialog Johanns mit einem alten Hofrat, der Grillparzer zum Verwechseln ähnlich sieht, wird den Deutschen

50 Zur Rolle Schreyvogls als Theaterautor in der Zwischenkriegszeit und zur Rezeption seiner Habsburgerdramen vgl. Pyrah, The Burgtheater and Austrian Identity (wie Anm. 6), S. $155-162$.

51 Friedrich Schreyvogl, Johann Orth. Österreichische Ballade. Wien 1928, S. 56 .

52 Ebd., S. 57.

53 Ebd.

54 Friedrich Schreyvogl, Habsburgerlegende. Berlin, Wien, Leipzig 1933, S. 160.

55 Ebd., S. 76.

56 Ebd., S. 20.

284 Roland Innerhofer 
der Vorzug zugeschrieben, Träumer zu sein. ${ }^{57}$ Als solche sind aber die Österreicher die besseren Deutschen. Wenn auch in der Gegenwart der Verlust der deutschen Mitte mit der Entwertung der traditionellen Werte einhergeht, ${ }^{58}$ gilt doch die Maxime, nach der Geschichte »im Hirn « ${ }^{59}$ entsteht ${ }^{60}$ Die im "Turm" handlungsbestimmende Aporie, dass der Geist die Geschichte lenken soll, aber in ihr die brutalen Machtpolitiker siegen, erscheint bei Schreyvogl als überwunden. Langfristig wird ein Triumph der geistigen Werte in Aussicht gestellt.

Anders als Schreyvogl verzichtet Hofmannsthal im "Turm« auf das Konzept der Nation als einigendes Band der Gemeinschaft. Während jener die Form der Legende übernimmt, um Johann als Märtyrer und Garant eines kommenden Reiches zu inszenieren, wird bei diesem die Gattung der Legende ausgehöhlt. Sigismund ist in der Bühnenfassung ein Märtyrer, der nicht weiß und von dem man nicht weiß, wofür er stirbt. Damit wird sowohl die Märtyrerfigur als auch die Legendenform fragwürdig.

\section{Fazit}

Im Vergleich zur österreichischen Dramatik der Vorkriegszeit fällt in den meisten besprochenen Texten eine Tendenz zur Verschiebung der Konflikte aus dem Individuellen ins Gesellschaftliche auf. Ihre Grundfrage lautet: Wie ist legitime Herrschaft nach der Beseitigung der Monarchie möglich?

Mit dem Ersten Weltkrieg sind sämtliche materiellen und ideellen Werte in Misskredit geraten, was Hofmannsthal, wie viele seiner schreibenden Zeitgenossen, nicht als Befreiung, sondern als Trauma erlebte. Die Angst vor dem Wertevakuum verbindet sich bei Hofmannsthal wie bei anderen konservativen Autoren mit der Angst vor dem Bolschewismus. In der Figur des Olivier zeichnet Hofmannsthal den Bolschewisten

57 Ebd., S. 76.

58 Ebd., S. 123.

59 Ebd., S. 35

60 Im Fall Schreyvogls stimmen die Ideen, welche die Geschichte bestimmen, weitgehend mit den jeweils herrschenden Ideologien des Ständestaates, der Austrofaschisten und später der Nationalsozialisten überein, insofern erscheinen sie als mit ihrer gewaltsamen Verwirklichung kompatibel. 
als Profiteur dieses Machtvakuums, der das Fatum als Alibi für seine Machtgelüste benutzt, zugleich aber nichts anderes als das Produkt solcher Fatalität ist.

Der Herrschaft materieller Gewalt, als die Hofmannsthal und andere die revolutionären Umbrüche in der Folge des Ersten Weltkrieges erleben, stellt Hofmannsthal die Erfindung einer Tradition ${ }^{61}$ entgegen, die eine legitime Ordnung verbürgen soll. Diese Tradition ist ein geistiges Österreich, das in Anknüpfung an das Heilige Römische Reich eine autoritäre Staatsführung nach dem Ende der Monarchie garantieren soll. Die Einheit eines solchen Reiches ist nicht national im völkischen Sinn, sondern beruht auf der Verbindlichkeit der Sprache, deren Gültigkeit durch auserwählte Dichter garantiert wird. Dieses in den essayistischen Arbeiten entworfene Programm nimmt aber die Endfassung des Turm in der dramatischen Performanz zurück.

Nachdem die Autorität eines politisch-theologisch begründeten Königtums spätestens im Ersten Weltkrieg endgültig diskreditiert worden war, traten Konzepte der Führerschaft in den Vordergrund, deren Gültigkeit und Anspruch auf Loyalität nicht 'gottgegeben sind, sondern von einer Anhängerschaft abhängen und erworben werden müssen. In dieser unsicheren Situation treten konservative und restaurative Staatskonzepte in Konkurrenz zu neuen Formen charismatischer Führung, die aus revolutionären Prozessen hervorgehen und sich auf moderne Formen der Propaganda stützen. Hofmannsthals Drama zeigt dabei die Fragilität charismatischer Herrschaft, deren Subjekt sich Alexander Mionskowki zufolge "als Hazardeur und Possenreißer und nur im Idealfall als >Verantwortungsethiker erweisen kann und zudem zwecks Machtsicherung auf ein Andauern oder ständiges Wiederkehren von krisenhaften bzw. außeralltäglichen Zuständen angewiesen ist. ${ }^{62}$ Hofmannsthal bringt, wie Mionskowski zeigt, in der Bühnenfassung des »Turm« neben dem traditionalen und dem charismatischen Max Webers dritten Typus der Herrschaftslegitimierung ins Spiel: den rationalen. ${ }^{63}$ Der Begriff der Rationalität betrifft im "Turm" aber nicht die Ziele, sondern ist rein in-

61 Vgl. Jaques Le Rider, Von der Erfindung einer Tradition zur konservativen Revolution. In: Ders., Hugo von Hofmannsthal. Historismus und Moderne in der Literatur der Jahrhundertwende. Aus dem Franz. von Leopold Federmair. Wien, Köln, Weimar 1997, S. 253-285.

62 Mionskowski, Souveränität als Mythos (wie Anm. 22), S. 383.

63 Vgl. ebd., S. 473. 
strumentell gemeint: Er bezieht sich lediglich auf den effizienten Einsatz von Gewalt, um die Macht des selbsternannten Volksvertreters Olivier zu sichern. ${ }^{64}$ Anders als Werfels Juarez, dessen Macht sich auf breite Zustimmung im Volk stützt, kann Olivier, um die Volksmassen zu mobilisieren, nicht auf die Inszenierung des Charismas, auf das (gefälschte) Bild des charismatischen Herrschers als Kollektiv-Symbol verzichten. Der charismatische Führer wird zum Komödianten. Wenn, wie Susanne Götz mit einigem Recht argumentiert, im »Turm« trotz aller Anstrengung »eines aufwendigen Abarbeitens an [...] der Calderon-Vorlage« "der groß angelegte Versuch einer Synthese mißlingt« und der Text »sich mit dem Nebeneinander von Disparatem zufrieden geben muß ${ }^{65}$ so ist das nicht als Manko zu bewerten. Der "Relativismus", die "ironische Distanz" und die »ethische Ironie", die Mathias Mayer dem »Schwierigen" als Kommentar auf die "Auflösung der Ordnung" in Folge des Ersten Weltkriegs attestiert, ${ }^{66}$ liegen, bei aller Düsternis seiner "resignativen Geschichtsdeutung «"67, auch dem »Turm» zugrunde.

Vor dem katholischen Hintergrund, vor dem sich viele Dramen österreichischer Zwischenkriegszeitautoren profilieren, ist Legitimität an sichtbare Symbole, Gesten und Rituale: an Theatralität geknüpft. Damit eignete sich das Drama in besonderem Maße dazu, die Frage nach der Führerschaft nicht nur darzustellen, sondern auch performativ vorzuführen. So leuchteten zahlreiche österreichische Dramen der Zwischenkriegszeit Auftritte neuer Führergestalten aus und führten damit eine Restitution von Autorität und staatlicher Ordnung vor. Hofmannsthals spätes Drama hebt sich von den Werken seiner konservativen Mitstreiter ab, indem es die Funktionsweise solcher Figuren und Vorgänge kritisch reflektiert und auf der Aporie neuer Führungsformen beharrt. Schmerzhaft kündigt sich hier der endgültige Abschied vom Konzept

64 Severin Perrig stellt in Hofmannsthals späten Dramen ein »mehr oder weniger selbständiges Agieren der Gewalt" fest, die einen ambivalenten Eindruck zwischen Schrecken und Faszination erzeuge. Severin Perrig, Hugo von Hofmannsthal und die Zwanziger Jahre. Eine Studie zur späten Orientierungskrise. Frankfurt a.M. 1994, S. 183f. In der Darstellung von Oliviers Gewaltherrschaft dominiert allerdings deutlich eine Stimmung düsterer Aussichtslosigkeit.

65 Susanne Götz, Bettler des Wortes. Irritationen des Dramatischen bei Sorge, Hofmannsthal und Horváth. Frankfurt a.M. 1998, S. 152.

66 Mathias Mayer, Der Erste Weltkrieg und die literarische Ethik. Historische und systematische Perspektiven. München 2010 (Ethik - Text - Kultur 4), S. 179.

67 Ebd. 
uneingeschränkter Souveränität, sei diese als geistige oder politische Macht verstanden, und ihr Umkippen in die totalitäre Diktatur an. Die Überwindung der Dichotomie von absoluter Souveränität vs. restloser Auslöschung des Subjekts und die daraus resultierenden Möglichkeiten teilsouveränen politischen Handelns sind Perspektiven, die »Der Turm« wenn überhaupt bloß ex negativo eröffnet. 